\title{
Prediction of response to pemetrexed in non-small-cell lung cancer with immunohistochemical phenotyping based on gene expression profiles
}

S. Visser ${ }^{1,2,3}$, J. Hou ${ }^{4}$, K. Bezemer ${ }^{2}$, L. L. de Vogel ${ }^{5}$, J. P. J. J. Hegmans² ${ }^{2}$ B. H. Stricker ${ }^{3}$, S. Philipsen ${ }^{4}$ and J. G. J. V. Aerts ${ }^{1,2^{*}}$

\begin{abstract}
Background: Palliative pemetrexed-based chemotherapy remains a standard of care treatment for the majority of patients with advanced non-squamous non-small-cell lung cancer (NSCLC). Currently, no predictive markers for pemetrexed treatment are available.

Methods: Resected tumour samples from pemetrexed-naïve NSCLC patients were collected. Gene expression profiling with respect to predicted sensitivity to pemetrexed classified predicted responders (60\%) and nonresponders (40\%) based on differentially expressed genes encoding for pemetrexed target enzymes. Genes showing a strong correlation with these target genes were selected for measurement of corresponding protein expressions by immunohistochemical $(\mathrm{HC})$ staining. A semi-quantitative $\mathrm{IHC}$ scoring method was applied to construct a prediction model for response to pemetrexed. A retrospective cohort of patients with advanced NSCLC treated with first-line pemetrexed-based chemotherapy was used for external validation.

Results: From ninety-one patients resected tumour samples were collected. The majority of patients had early or locally advanced NSCLC (96.3\%). Gene expression profiling revealed five markers, which mRNA levels strongly correlated to pemetrexed target genes mRNA levels: TPX2, CPA3, EZH2, MCM2 and TOP2A. Of 63 (69\%) patients $\mathrm{IHC}$ staining scores of these markers were obtained, which significantly differed between predicted non-responders and responders $(P<0.05)$. The optimized prediction model included EZH2 $(\mathrm{OR}=0.56,95 \% \mathrm{Cl} 0.35-0.90)$ and TPX2 $(\mathrm{OR}=0.55,95 \% \mathrm{Cl} 0.30-1.01)$. The model had a sensitivity of $86.8 \%$, specificity of $63.6 \%$ and showed a good ability to distinct between responders and non-responders (C-index 0.86 ).

In the external study population $(N=23)$ the majority of patients had metastatic NSCLC (95.7\%). Partial response (PR) was established in $26.1 \%$. The sensitivity decreased drastically to $33.3 \%$, with a specificity of $82.4 \%$ and a C-index of 0.73 .

Conclusions: Using external validation this prediction model with $\mathrm{IHC}$ staining of target enzyme correlated markers showed a good discrimination, but lacked sensitivity. The role of $\mathrm{HC}$ markers as response predictors for pemetrexed in clinical practice remains questionable.
\end{abstract}

Keywords: NSCLC, Pemetrexed, Response prediction, Gene-expression profiling, Immunohistochemistry

* Correspondence: j.aerts@erasmusmc.nl

${ }^{1}$ Department of Pulmonary Medicine, Amphia Hospital, Breda, the Netherlands

${ }^{2}$ Department of Pulmonary Medicine, Erasmus MC Cancer Institute, P.O. Box

2040, 3000, CA, Rotterdam, The Netherlands

Full list of author information is available at the end of the article

(c) The Author(s). 2019 Open Access This article is distributed under the terms of the Creative Commons Attribution 4.0 International License (http://creativecommons.org/licenses/by/4.0/), which permits unrestricted use, distribution, and reproduction in any medium, provided you give appropriate credit to the original author(s) and the source, provide a link to the Creative Commons license, and indicate if changes were made. The Creative Commons Public Domain Dedication waiver (http://creativecommons.org/publicdomain/zero/1.0/) applies to the data made available in this article, unless otherwise stated. 


\section{Background}

In the management of advanced non-small cell lung cancer (NSCLC) systemic treatment options are rapidly expanding with the increasing use of molecular targeted agents and immunotherapy [1-4]. One of the most important therapeutic advances has been the identification of predictive molecular markers to guide patient selection for frontline treatment with these agents, like sensitizing mutations within the EGFR gene to EGFR tyrosine kinase inhibitors and protein PD-L1 overexpression to anti PD-(L)1 checkpoint inhibitors [1, 2, 5]. Despite the changing treatment landscape with increasing use of molecular targeted agents and immunotherapy, pemetrexed-based chemotherapy is still widely used as standard treatment in patients with advanced nonsquamous non-small-cell lung cancer $[6,7]$. Unfortunately, to date useful biomarkers predicting response to this treatment regimen are lacking.

Pemetrexed treatment shows a variable clinical efficacy apparently dependent on the histologic subtype of lung cancer. Clinical trials demonstrated efficacy of pemetrexed in nonsquamous NSCLC while efficacy was worse in squamous NSCLC and small-cell lung cancer [8-10]. However, tumour response to pemetrexed also differs significantly between patients with similar histology $[9,11]$. In patients treated with pemetrexed monotherapy, the response rate to pemetrexed was evidently different between histological subtypes but low in both patients with squamous and non-squamous NSCLC $(2.8 \%$ vs. $11.5 \%)$ [10]. In this study pemetrexed was administered as second-line treatment and patients with poor ECOG performance score were included. Although the response rate to pemetrexed was significantly higher in patients with non-squamous versus squamous NSCLC in the first-line pivotal trial [9], still more than $20 \%$ of patients with squamous NSCLC had a response to pemetrexed while the response rate was merely $\sim 30 \%$ in patients with non-squamous histology [11]. These findings highlight the need for predictive molecular markers for pemetrexed-based treatment.

The main determinant of pemetrexed responsiveness is thought to be the level of expression of thymidylate synthase (TS), the primary intracellular target enzyme of pemetrexed $[12,13]$. Overexpression of TS mRNA has been correlated with reduced sensitivity to pemetrexed in vitro, [14-17] and with worse clinical outcomes in patients treated with pemetrexed [18]. Moreover, the abundance of TS expression is higher in squamous cell NSCLC than in other histologic subtypes, $[19,20]$ which constitutes the biological hypothesis behind the superior efficacy of pemetrexed in non-squamous NSCLC. However, in clinical practice the relationship between protein expression levels of TS, measured by immunohistochemistry (IHC) methods, and the clinical efficacy of pemetrexed remains controversial [21-25].
Our study group earlier presented an approach to implement a more refined molecular classification of NSCLC subtypes based on gene expression profiles independent of histology [26]. Furthermore, response to pemetrexed was predicted based on expression of genes encoding different pemetrexed target enzymes including but not limited to TS, and expression signatures of correlated genes were identified. In the current study, we explore whether these differential gene expression profiles between responders and non-responders can be used to define a prediction model based on IHC scores of selected molecular markers.

\section{Methods}

\section{Training cohort}

Tumour samples from pemetrexed-naïve, NSCLC patients who had undergone curative surgical resection at the Erasmus Medical Center (Rotterdam) between 1992 and 2004 were used. A detailed description of tissue collection, microarray preparation and data processing, the derivation of a gene-expression based predictive algorithm for tumour response and the identification of pemetrexed resistance-associated genes has been previously described [26]. In short, the predictive algorithm predicted tumour response based on the expression difference between internal reference genes and pemetrexed target genes TS, dihydrofolate reductase (DHFR) and glycinamide ribonucleotide formyltransferase (GARFT). Using percentile-rank based target gene expression levels relative to the internal reference genes, patients were stratified as predicted responders $( \pm 60 \%)$ and non-responders $( \pm 40 \%)$. Subsequently, significance analysis of microarray identified differentially expressed genes between these classified groups [27]. A minimized signature containing 25 genes performed optimally in predicting pemetrexed response (Additional file 1: Table S1). For the current study, we selected molecular markers from this signature if they showed a strong correlation with the gene expression of TS and if IHC stainings for these markers were commercially available. Written informed consent was obtained from all these patients. The study was conducted in accordance with the REMARK guidelines [28].

\section{Validation cohort}

In order to externally validate the model, we obtained formalin-fixed paraffin-embedded pre-treatment biopsies of a retrospective cohort of patients newly diagnosed with advanced stage (IIIB/IV) NSCLC in a large teaching hospital (Amphia hospital, Breda, the Netherlands) between January 2007 and December 2010. Patients were eligible for enrolment if they had received $\geq 2$ cycles of platinum-combined pemetrexed chemotherapy as first-line treatment. Medical charts and radiological imaging data were reviewed to collect information regarding 
socio demographics, tumour histology, ECOG performance status, treatment and observed tumour response (RECIST 1.1). Patients with early stage (IA-IIB) or locally advanced (IIIA) disease, (neo)adjuvant chemotherapy, combination treatment with bevacizumab and without tissue samples from primary tumour or (lymph node) metastasis were excluded.

\section{Tissue microarray analysis and immunohistochemistry}

The tissue microarrays (TMAs) were composed of 68 of the 91 tumour tissues, in triplicate, from the Erasmus MC patient cohort used for the expression microarray analyses. TMA blocks containing $0.6 \mathrm{~mm}$ cores of formalin-fixed paraffin-embedded tumours were cut and antigen retrieval was performed by a 20 -min incubation at $95{ }^{\circ} \mathrm{C}$ using Trisethylenediaminetetraacetic acid buffer (Klinipath, Duiven, The Netherlands). Subsequently, TMAs were stained with primary antihuman antibodies of the selected candidate markers: EZH2, TOP2A, TPX2, MCM2 and CPA3 (Additional file 2: Table S2). For each TMA multicontrol stainings were performed using a combination of tissues (liver, pancreas, tonsil, colon and appendix). The slides were stained and processed in the Ventana Benchmark ULTRA strainers, using DAB as substrate and Hematoxylin as counterstain. Tumour tissues from the validation group were equally handled, except that the tumour samples were cut in $0.4 \mathrm{~mm}$ instead of $0.6 \mathrm{~mm}$ cores for TMA blocks.

\section{Immunohistochemical staining score}

A semi-quantitative scoring method was applied to classify the intensity and quantity of IHC staining of candidate markers. The quantity score was defined as: 1: 0 30\%; $2: 30-60 \% ; 3: 60-100 \%$. The intensity score was defined as: 0 (negative), no appreciable staining in the tumour cells; 1 (weak), barely detectable cytoplasmic/ membranous or nuclear staining of tumour cells; 2 (moderate), readily appreciable staining of tumour cytoplasm/nucleus; 3 (strong), strong staining obscuring nucleus/cytoplasm of tumour cells. Multiplying quantity and intensity score yielded a total score with a range between 0 and 9. TMAs from the training group were evaluated and scored for protein expression simultaneously by K.B. and J.P.H. Samples were individually discussed until consensus was reached. For the validation group, TMA evaluation and protein staining quantification were performed independently by K.B. and S.V.

\section{Statistical analyses}

Sociodemographic and disease- and treatment-related variables were described for all patients who were included in this study and were compared between the training and validation group. We used the independentsamples $t$-test and the $\chi^{2}$-test or Fisher's exact test for continuous and categorical variables respectively. Degree of agreement on quantity and intensity scores of the different IHC stainings was evaluated using weighted linear Cohen's kappa scores ( ) in the validation group. Degree of agreement was determined according to widely used scale described by Landis and Koch [29]. As IHC staining scores from the training group were obtained by discussion, and thus not independently, no interobserver agreement could be calculated.

Pearson correlation coefficients $(\rho)$ were calculated between gene expression of the candidate markers and TS in the training group and subsequently between the gene expression of those markers and their associated protein expressions. Using the described prediction algorithm of response to pemetrexed, patients from the training group were divided in predicted responders and non-responders. In the training group, we compared gene and protein expression from selected molecular markers between predicted responders and non-responders.

Logistic regression with dependent variable predicted tumour response by gene expression signature was applied to the training cohort to build a prediction model with the IHC staining scores of selected molecular markers as independent variables. Optimized model derivation was performed using purposeful selection by stepwise in- and exclusion of molecular markers [30]. Univariable logistic regression identified molecular markers associated with predicted tumour response. We specified a priori that molecular markers with $p<0.2$ on univariable analysis would be candidate variables for multivariable logistic regression model. In the iterative process of variable selection, variables were removed from the model if they were non-significant and not a confounder. We used backward selection with a $p$-value $<0.05$ and/or change of effect size of (an)other included variable(s) $>20 \%$ to remain in the model. The fit of a reduced model versus full model was compared with the likelihood ratio test (LRT), following a chi-squared distribution. Subsequently, the model was externally validated in the validation cohort. In both cohorts, the model performance of the derived model was assessed by examining the predictive classification accuracy and discriminatory ability (C-index). A C-index of 1.0 would indicate perfect discrimination, whereas a $\mathrm{C}$-index of 0.5 indicates total absence of discrimination. All statistical analyses were performed with the use of SPSS, version 24.0 (IBM Corporation, Armonk, NY).

\section{Results}

The selection of patients in both training and validation cohort is depicted in Fig. 1. Of the 91 surgically resected samples of the primary tumour, 68 (74.7\%) samples were suitable for further processing into TMAs. Since samples of five patients could not be used due to insufficient 


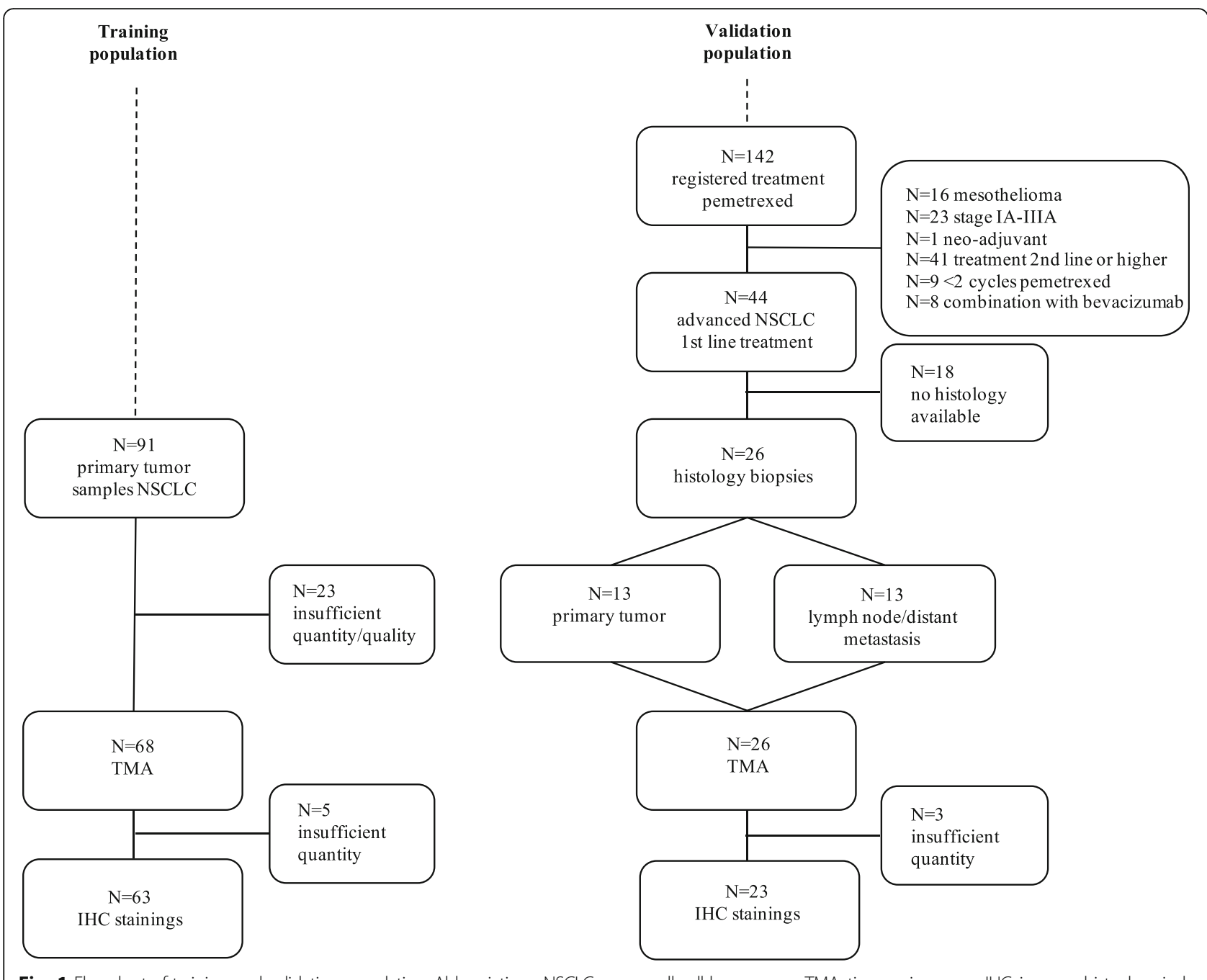

Fig. 1 Flowchart of training and validation population. Abbreviations: NSCLC, non-small-cell lung cancer; TMA, tissue micro array; IHC, immunohistochemical

TMA material, the training cohort ultimately consisted of 63 patients whose samples were prepared with additional IHC stainings. For validation, 44 of 142 (31\%) patients who received pemetrexed had advanced stage NSCLC treated with $\geq 2$ cycles first-line treatment with platinum-combined pemetrexed, excluding combinations with bevacizumab. Of these patients, 18 (40.9\%) were excluded from further analysis because their diagnosis was cytology-based and no histologic biopsy was obtained. Histology samples were retrieved from primary tumour $(N=13)$ or lymph nodes or distant metastases $(N=13)$. Three patients had insufficient tumour material available for additional IHC staining and therefore the validation cohort consisted of 23 patients.

\section{Patient characteristics}

Patient and treatment characteristics are shown in Table 1 . In the training cohort 46 patients (73.0\%) were male compared to $10(43.5 \%)$ in the validation cohort.
Eighteen patients (28.6\%) had squamous NSCLC in the training cohort while in the validation cohort only patients were included with non-squamous histology. The majority of patients $(95.2 \%)$ in the training group had early stage NSCLC opposed to all patients with advanced disease stage in the validation group. No data were available with regard to the ECOG performance score of the patients in the training cohort. In the validation cohort, $21.7 \%$ of the patients had a performance score of 2 . Corresponding to the differences in disease stage between the cohorts, all patients in the training cohort underwent surgical resection in contrast to palliative pemetrexed-based chemotherapy in the validation cohort. Patients in the training cohort had worse overall survival compared to the validation cohort ( 4.5 months vs. 28 months).

\section{Selection molecular markers}

Of the 25-genes containing optimized gene expression signature predicting response to pemetrexed in the 
Table 1 Characteristics of patients in the training population and the validation population

\begin{tabular}{|c|c|c|}
\hline & $\begin{array}{l}\text { Training cohort } \\
(N=63)\end{array}$ & $\begin{array}{l}\text { Validation cohort } \\
(N=23)\end{array}$ \\
\hline Age, mean (SD) & $61.9( \pm 10.7)$ & $58.7( \pm 8.7)$ \\
\hline Gender, male & $46(73.0)$ & $10(43.5)$ \\
\hline \multicolumn{3}{|l|}{ Smoking status } \\
\hline Never smoker & $1(1.6)$ & $1(4.3)$ \\
\hline Ever smoker & $31(49.2)$ & $21(91.3)$ \\
\hline Unknown & $31(49.2)$ & $1(4.3)$ \\
\hline \multicolumn{3}{|l|}{ ECOG performance score } \\
\hline 0 or 1 & & $17(73.9)$ \\
\hline 2 & & $5(21.7)$ \\
\hline Unknown & $63(100)$ & $1(4.3)$ \\
\hline \multicolumn{3}{|l|}{ Histology } \\
\hline$A D C$ & 18 (28.6) & $21(91.3)$ \\
\hline LCC & $24(38.1)$ & $2(8.7)$ \\
\hline SCC & $15(23.8)$ & \\
\hline Other & $6(9.5)$ & \\
\hline \multicolumn{3}{|l|}{ Tumor stage } \\
\hline $\mid A-\| B$ & $56(88.9)$ & \\
\hline$\| \mathrm{A}$ & $4(6.3)$ & \\
\hline$\| \mathrm{II}$ & & $1(4.3)$ \\
\hline IV & $3(4.8)$ & $22(95.7)$ \\
\hline \multicolumn{3}{|l|}{ Treatment } \\
\hline Surgery & $63(100)$ & \\
\hline CISPEM & & $18(78.3)$ \\
\hline CARPEM & & $5(21.7)$ \\
\hline $\begin{array}{l}\text { No. cycles chemotherapy, } \\
\text { median (IQR) }\end{array}$ & & $3(3-4)$ \\
\hline \multicolumn{3}{|l|}{ Treatment effect } \\
\hline PR & & $6(26.1)$ \\
\hline SD & & $7(30.4)$ \\
\hline PD & & $10(43.5)$ \\
\hline OS, median (IQR) & $28.0(10.0-67.6)$ & $4.5(3.2-7.3)$ \\
\hline
\end{tabular}

Data are expressed as numbers (\%) unless stated otherwise. Abbreviations: $S D$ standard deviation, $A D C$ adenocarcinoma, $L C C$ large cell carcinoma, SCC squamous cell carcinoma, CISPEM cisplatin combined with pemetrexed, CARPEM carboplatin combined with pemetrexed, OS overall survival, IQR interquartile range, $P R$ partial response, $S D$ stable disease, $P D$ progressive disease

training group, five molecular markers were selected based on their correlation with the gene expression level of target genes (TS, DHFR, GARFT) and the commercial availability of corresponding IHC stainings: Enhancer of zeste homolog 2 (EZH2), Topoisomerase II Alpha (TOP2A), Microtubule Nucleation Factor (TPX2), Carboxypeptidase A3 (CPA3) and Minichromosome Maintenance Complex Component 2 (MCM2). All markers showed a positive correlation to the mRNA level of TS (EZH2, $\rho=0.732$; MCM2, $\rho=0.804$; TOP2A, $\rho=0.814$;
TPX2, $\rho=0.825)$, except for CPA3 which was negatively correlated $(\rho=-0.467)$ (Additional file 4: Figure S1). The correlation of gene mRNA level with their corresponding IHC staining score had a range between 0.303 (CPA3) and 0.578 (EZH2) (Additional file 5: Figure S2).

The IHC stainings of the same markers were applied to the TMAs of the samples of patients in the validation cohort. The strength of agreement between the observers with regard to the intensity score ranged between 휄 = 0.515 (CPA3) and 훽 = 1 (MCM2), and with regard to the quantity score between 휄 $=0.547(\mathrm{TPX} 2)$ and 휄 $=0.851$ (CPA3). Weighted kappa values of IHC staining scores are outlined in Additional file 3: Table S3.

Of all selected markers, both mRNA levels and IHC staining scores were significantly higher in predicted non-responders than responders in the training group, except for CPA which mRNA level and IHC staining score was significantly lower in non-responders compared to responders. These results are depicted in Fig. 2.

\section{Model derivation}

The model coefficients and odds ratios (OR) with corresponding $95 \%$ confidence intervals $(\mathrm{CI})$ of the prediction model with dependent variable tumour response to pemetrexed using univariable and multivariable logistic regression analyses are presented in Table 2 . Univariable analyses of the relationship between the IHC staining scores of the selected markers and the gene expression based predicted tumour response to pemetrexed were performed using the training cohort. Higher IHC staining scores of all markers were significantly associated with worse predicted tumour response, except for CPA3 which repeatedly showed the reverse association compared to the other markers. Using multivariable analysis, only a higher IHC staining score of EZH2 was significantly related with a worse predicted tumour response to pemetrexed (OR 0.56, 95\% CI 0.35-0.90; $P=0.015$ ). The staining scores of all other markers failed to demonstrate a significant association on multivariable analysis. Although IHC staining score of TPX2 was not significantly associated with tumour response in the multivariable model (OR $0.55,95 \%$ CI $0.30-1.01 ; P=0.054$ ), this variable was still included in the final optimized prediction model. Removal of this variable led to a significantly reduced model fit $(P<0.001)$.

\section{Model performance and validation}

In Table 3 the different test characteristics describing the performance of the model in the training and validation cohort are shown. In the training cohort, 38 patients were predicted responders (63.3\%) by gene expression profiling and $86.8 \%$ (33 of 38 ) were correctly classified responders by the prediction model (sensitivity $86.8 \%, 95 \%$ CI 71.9 95.6). Fourteen patients were predicted non-responders 


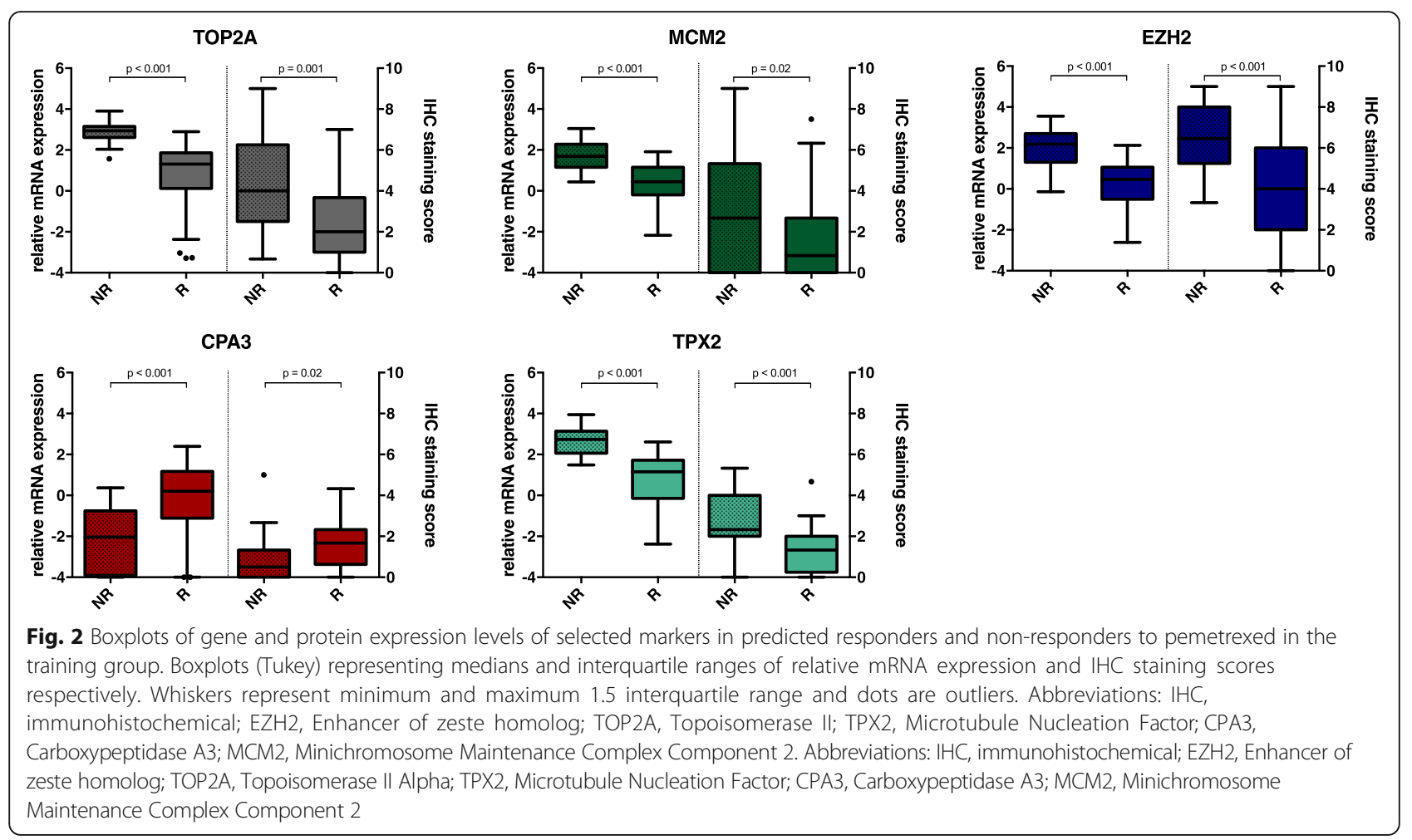

(36.7\%) by gene expression profiling while $63.6 \%$ (14 of 22) were correctly classified as non-responders by the model (specificity 63.6\%, 95\% CI 40.7-82.8).

In the validation cohort, the same classification by the prediction model was applied, however an actual tumour response was obtained as these patients were treated with pemetrexed. The response rate was $26.1 \%$ and therefore the prevalence of response was substantially lower than in the training cohort. Of the six patients who experienced a partial response, two patients were correctly classified by the prediction model resulting in a sensitivity of $33.3 \%$ (95\% CI 4.3-77.7). Herewith, the sensitivity in this cohort is significantly worse than the sensitivity in the training cohort (Fisher's exact test, $P=$ 0.011). The positive predictive value (PPV) also decreased substantially. If we classified both patients with a partial response and stable disease as responders (56.5\%), performance characteristics of the model declined dramatically (Table 3 ).

The ROC curve showed a C-index of 0.86 (95\% CI 0.77-0.96) in the training cohort, representing a good discriminatory performance. The $\mathrm{C}$-index decreased to 0.73 (95\% CI $0.52-0.93$ ) if the prediction model was applied to the validation cohort (Fig. 3).

\section{Discussion}

Currently, the profit of and need for molecular markers to select therapy for individual patients is increasingly recognized. The last several years, treatment of advanced NSCLC has become obviously more complex and therefore tools to choose therapies that are most likely to benefit patients are required. Indeed the registration of

Table 2 Prediction model derivation to predict tumor response using $\mathrm{IHC}$ staining scores of selected molecular markers in training group $(N=63)$

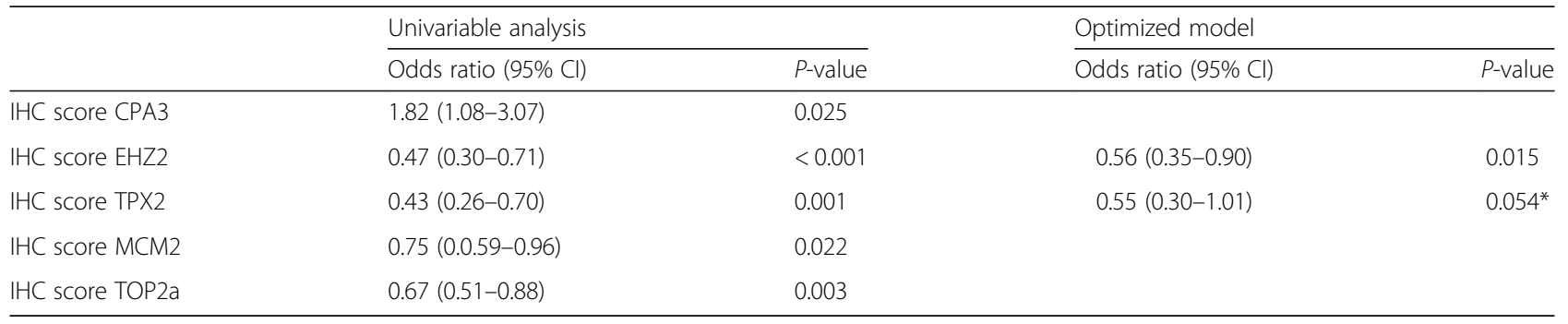

*Model fit was significantly worse (based on difference - 2 Log Likelihood) if TPX2 was excluded. Abbreviations: $\mathrm{Cl}$ confidence interval, EZH2 Enhancer of zeste homolog, TOP2A Topoisomerase II Alpha, TPX2 Microtubule Nucleation Factor, CPA3 Carboxypeptidase A3, MCM2 Minichromosome Maintenance Complex Component 2 
Table 3 Conditional and post-test probability performance of the IHC based prediction model in the training and validation cohort

\begin{tabular}{llll}
\hline & Training cohort & Validation cohort Responder: PR & Validation cohort Responder: PR + SD \\
\hline Prevalence (responder) & $63.3(49.9-75.4)$ & $26.1(10.2-48.4)$ & $56.5(34.5-76.8)$ \\
Sensitivity & $86.8(71.9-95.6)$ & $33.3(4.3-77.7)$ & $15.4(1.9-45.5)$ \\
Specificity & $63.6(40.7-82.8)$ & $82.4(56.6-96-2)$ & $70.0(34.6-93.3)$ \\
LR+ (weighted by prevalence) & $2.39(1.36-4.21)$ & $1.89(0.41-8.71)$ & $0.51(0.10-2.51)$ \\
LR- (weighted by prevalence) & $0.21(0.09-0.50)$ & $0.81(0.44-1.49)$ & $1.21(0.76-1.93)$ \\
PPV & $80.5(70.1-87.9)$ & $40.0(12.6-75.5)$ & $40.0(12.0-76.5)$ \\
NPV & $73.7(53.8-87.1)$ & $77.8(65.6-86.5)$ & $38.9(28.5-50.4)$ \\
\hline
\end{tabular}

Data are expressed as percentages, except LR+ and LR- (odds), with 95\% confidence intervals. Abbreviations: $L R+$ positive likelihood ratio, $L R$ - negative likelihood ratio, $P P V$ positive predictive value, NPV negative predictive value

new therapeutic agents as frontline therapy is accompanied by selective markers. For patients with EGFR mutation-positive, ALK rearrangement-positive or ROS1 rearrangement positive tumours first-line molecular targeted tyrosine kinase inhibitors are recommended, while patients with high PD-L1 expression in the tumour are suitable for pembrolizumab first-line treatment [6]. Despite the wide implementation of molecular markers, the administration of chemotherapy in NSCLC patients is still solely based on histology even though its capacity to predict response has been proved to be suboptimal.

In an earlier study, we demonstrated an approach to predict response to pemetrexed based on the use of gene expression profiles [26]. Samples of different histological subtypes including squamous NSCLC were used. Prediction of response to pemetrexed based on gene expression profiling of its target enzymes, failed to show the expected disadvantage for the squamous cell histological subtype and these results therefore challenge the restricted use of pemetrexed in non-squamous NSCLC. In the present study, we developed a prediction model using immunohistochemistry scores of selected candidate genes (EZH2 and TPX2) from the gene expression signature predicting pemetrexed response. In the training group, the use of the model resulted in good performance characteristics of the model in the sense of a high sensitivity and PPV. The model also showed the ability to discriminate well between responders and non-responders. Unfortunately, the results of the obtained model could not be validated when applied to an external cohort of patients treated with pemetrexed.

Although it is hypothesized that superior efficacy of pemetrexed in non-squamous over squamous NSCLC is related to the level of TS expression, multiple clinical studies failed to demonstrate the association between TS protein expression and clinical outcomes [22, 25]. This can be ascribed firstly to the fact that current semi-quantitative IHC methods might lack sensitivity required to measure protein expression of TS opposed to quantitative analysis of mRNA expression. And secondly, the biological significance of TS for pemetrexed responsiveness might be of less importance than other molecular processes, e.g. gene expression or amplification of other (target) genes. To overcome these limitations in the present study, we carefully selected

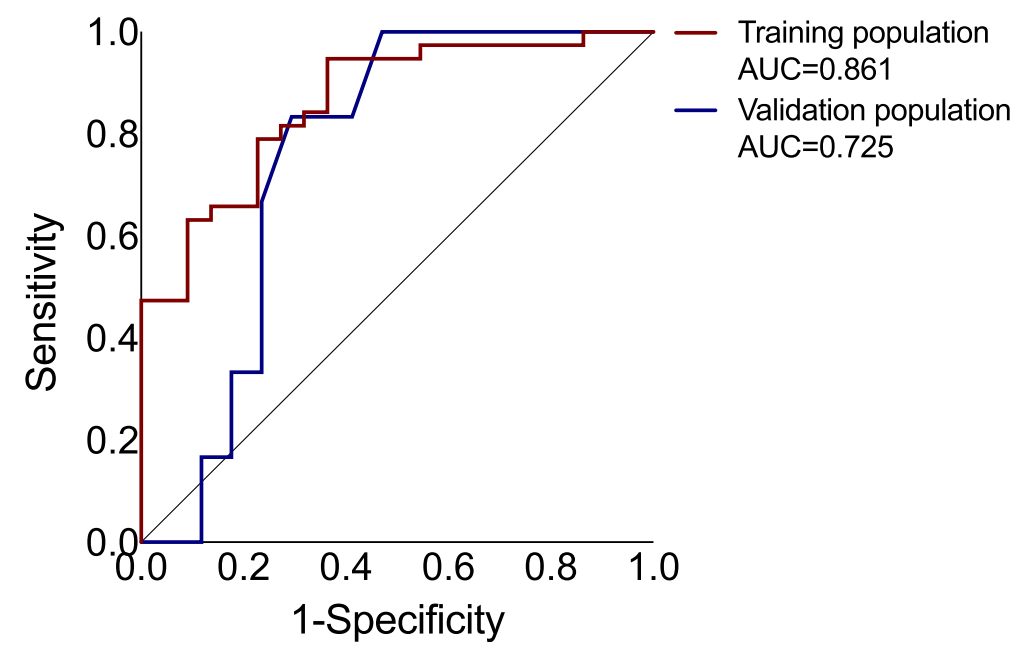

Fig. 3 Receiver operating characteristic curve showing model performance of two-protein expression prediction model in training and validation population. Diagonal line reflects total absence of discrimination ( $\cup \cup C=0.5)$. Abbreviations: $A \cup C$, area under the curve 
markers related to mRNA gene expression of TS but also to other target enzymes DHFR and GARFT.

In the literature, both EZH2 and TPX2 have been previously linked to survival in NSCLC. TPX2 is involved in key steps during mitotic events and increased expression has been associated with poor overall survival in NSCLC $[31,32]$. EZH2 epigenetically silences multiple genes involved in cell differentiation, growth and invasion. It is often overexpressed in NSCLC promoting cancer progression and a more aggressive tumour behaviour $[33,34]$. Downregulation of EZH2 has been associated with higher expression of oestrogen receptor and increased sensitivity to tamoxifen in advanced breast cancer patients [35]. Similarly, one can speculate that EZH2 might change the expression of genes related to responsiveness of pemetrexed through its ability to silence other genes. Although lack of a control arm precludes discrimination between a prognostic or predictive factor, we purposefully focused on radiological response rather than survival.

It is crucial to predict treatment effects for individual patients in order to avoid unnecessary toxicities and to offer alternative treatment options, with few false positives [36]. The clinical value of the derived model is probably limited as the sensitivity was poor in the validation group. Moreover, the low PPV makes the classifier not useful for clinical decision making, as many patients who are predicted responders will then actually undergo potentially harmful treatment with low chance of tumour response. The failure of the prediction model to adequately perform in the external patient population might be ascribed to an insufficient sensitivity of used IHC assays to measure significant differences in protein expression or a discrepancy between protein expression and protein activity. Additionally, spatiotemporal heterogeneity might have led to different intrinsic tumour properties in the validation group as these patients had advanced disease and in half of the cases tumour samples were obtained from lymph node or distant metastases. Finally, other factors might influence pemetrexed activity such as cell transport and intracellular formation of polyglutamate metabolites [37]. Our study was limited in the number of patients included, especially in the external validation.

cohort. We recognize that the differences in histology between the training and validation cohort is a major shortcoming. It was impossible to include patients with non-squamous NSCLC to the validation cohort, as selection was treatment-based and pemetrexed is only recommended in patients with nonsquamous NSCLC. For ALK and ROS1-rearrangement positive adenocarcinoma patients might experience more benefit to pemetrexedbased chemotherapy $[38,39]$, molecular characteristics would have been desirable. Unfortunately, those data were not available in our cohort, but the high number of smokers profoundly reduces the chance of rearrangements. Although response rates were in accordance with the literature, patients in the validation cohort experienced a very poor median overall survival of only 4.5 months. This can be partially explained by the presence of a substantial group of patients $(>20 \%)$ with a poor ECOG performance score and suboptimal treatment with carboplatin instead of cisplatin combination. Whether these patients appropriately represent the population with advanced NSCLC is therefore highly questionable and we cannot exclude their genetic profile to be different. However, given the results we do not expect that expanding the number of samples will lead to a clinically useful biomarker.

\section{Conclusion}

There remains an unmet need to identify biomarkers to select patients for standard pemetrexed-based treatment. Prediction of pemetrexed responsiveness with IHC stainings of markers correlated to TS and other target enzymes could not be validated using external validation. Future research focusing on metabolomics, pharmacokinetics and pharmacogenetics might offer new insights into tailoring therapy. Until a well-validated biomarker is identified, histology should remain the standard to select advanced NSCLC patients eligible for treatment with pemetrexed.

\section{Additional files}

Additional file 1: Table S1. Minimized signature for prediction of pemetrexed response. (DOCX $66 \mathrm{~kb}$ )

Additional file 2: Table S2. Antibodies used for immunohistochemical analyses. (DOCX $33 \mathrm{~kb}$ )

Additional file 3: Table S3. Interobserver agreement of the $\mathrm{HC}$ staining score with regard to tumour quantity and intensity of staining. (DOCX $39 \mathrm{~kb}$ )

Additional file 4: Figure S1. Scatter plots of gene expression levels of selected molecular markers and TS gene expression. Dot plots showing correlations between relative mRNA expression of TS and mRNA expression of TOP2A, MCM2, EZH2, CPA3, TPX2. Abbreviations: IHC, immunohistochemical; EZH2, Enhancer of zeste homolog; TOP2A, Topoisomerase II; TPX2, Microtubule Nucleation Factor; CPA3, Carboxypeptidase A3; MCM2, Minichromosome Maintenance Complex Component 2. (EPS $180 \mathrm{~kb}$ )

Additional file 5: Figure S2. Scatter plots of gene expression levels of selected molecular markers with their protein expression level and the associated correlations. Dot plots showing correlations between relative mRNA expression and IHC staining score of TOP2A, MCM2, EZH2, CPA3, TPX2. Abbreviations: IHC, immunohistochemical; EZH2, Enhancer of zeste homolog; TOP2A, Topoisomerase II; TPX2, Microtubule Nucleation Factor; CPA3, Carboxypeptidase A3; MCM2, Minichromosome Maintenance Complex Component 2. (EPS $176 \mathrm{~kb}$ )

\section{Abbreviations}

Cl: Confidence interval; CPA3: Carboxypeptidase A3; DHFR: Dihydrofolate reductase; EZH2: Enhancer of zeste homolog 2; GARFT: Glycinamide

ribonucleotide formyltransferase; IHC: Immunohistochemical; LRT: Likelihood ratio test; MCM2: Minichromosome Maintenance Complex Component 2; NSCLC: Non-small-cell lung cancer; OR: Odds ratio; PPV: Positive predictive value; TMA: Tissue microarrays; TOP2A: Topoisomerase II Alpha;

TPX2: Microtubule Nucleation Factor; TS: Thymidylate synthase 


\section{Acknowledgements}

Not applicable

\section{Funding}

This study was supported by ZonMw, the Netherlands [grant number 152001017]. The funding was used to perform the immunohistochemical stainings on the TMAs of the patients in the validation cohort. The funding body had no role in the design of this study and collection, analysis and interpretation of the data or in writing the manuscript.

\section{Availability of data and materials}

The microarray data of the training cohort are available at the Gene Expression Omnibus of the National Center for Biotechnology Information (GSE19188). https://www.ncbi.nlm.nih.gov/geo/query/acc.cgi?acc=GSE19188 The datasets used and/or analysed during the current study are available from the corresponding author on reasonable request.

\section{Authors' contributions}

$J A, B S$ and SV formulated the study design. SV, JH, KB, LV, JPH and SP performed the data acquisition. Scoring of the immunohistochemical stainings was done by SV, KB and JPH. SV and BS completed the statistical analyses. All authors were involved in manuscript preparation and editing. All authors read and approved the final manuscript.

\section{Ethics approval and consent to participate}

Written informed consent was obtained from all participants in the training cohort. Tissues were studied under an anonymous tissue protocol approved by the medical ethical committee of Erasmus University Medical Center. As data in the validation cohort were collected retrospectively, no approval by a medical research and ethics committee was necessary according to Dutch guidelines (https://english.ccmo.nl).

\section{Consent for publication}

Not applicable

\section{Competing interests}

Joachim Aerts has a consultant/advisory role with Eli Lilly and Company, Roche, Bristol-Myers Squibb, MSD and Boehringer Ingelheim. The other authors declare no conflicts of interest.

\section{Publisher's Note}

Springer Nature remains neutral with regard to jurisdictional claims in published maps and institutional affiliations.

\section{Author details}

'Department of Pulmonary Medicine, Amphia Hospital, Breda, the Netherlands. ${ }^{2}$ Department of Pulmonary Medicine, Erasmus MC Cancer Institute, P.O. Box 2040, 3000, CA, Rotterdam, The Netherlands. ${ }^{3}$ Department of Epidemiology, Erasmus MC, Rotterdam, the Netherlands. ${ }^{4}$ Department of Cell Biology, Erasmus MC, Rotterdam, the Netherlands. ${ }^{5}$ Department of Pathology, Erasmus MC, Rotterdam, the Netherlands.

Received: 15 August 2018 Accepted: 26 April 2019

Published online: 14 May 2019

\section{References}

1. Mok TS, Wu Y-L, Thongprasert S, Yang C-H, Chu D-T, Saijo N, et al. Gefitinib or carboplatin-paclitaxel in pulmonary adenocarcinoma. N Engl J Med. 2009;361:947-57. https://doi.org/10.1056/NEJMoa0810699.

2. Shaw AT, Kim D-W, Nakagawa K, Seto T, Crinó L, Ahn M-J, et al. Crizotinib versus chemotherapy in advanced ALK -positive lung Cancer. N Engl J Med. 2013;368:2385-94. https://doi.org/10.1056/NEJMoa1214886.

3. Reck M, Kaiser R, Mellemgaard A, Douillard J-Y, Orlov S, Krzakowski M, et al. Docetaxel plus nintedanib versus docetaxel plus placebo in patients with previously treated non-small-cell lung cancer (LUME-lung 1): a phase 3, double-blind, randomised controlled trial. Lancet Oncol. 2014;15:143-55. https://doi.org/10.1016/S1470-2045(13)70586-2.

4. Borghaei H, Paz-Ares L, Horn L, Spigel DR, Steins M, Ready NE, et al. Nivolumab versus docetaxel in advanced nonsquamous non-small-cell lung Cancer. N Engl J Med. 2015;373:1627-39. https://doi.org/10.1056/NEJMoa 1507643.
5. Reck M, Rodríguez-Abreu D, Robinson AG, Hui R, Csőszi T, Fülöp A, et al. Pembrolizumab versus chemotherapy for PD-L1-positive non-small-cell lung Cancer. N Engl J Med. 2016;375:1823-33. https://doi.org/10.1056/ NEJMoa1606774.

6. Hanna N, Johnson D, Temin S, Baker S, Brahmer J, Ellis PM, et al. Systemic therapy for stage IV non-small-cell lung Cancer: American Society of Clinical Oncology clinical practice guideline update. J Clin Oncol. 2017;/CO2017746065. https://doi.org/10.1200/JCO.2017.74.6065.

7. Novello S, Barlesi F, Califano R, Cufer T, Ekman S, Levra MG, et al. Metastatic non-small-cell lung cancer: ESMO clinical practice guidelines for diagnosis, treatment and follow-upt. Ann Oncol. 2016;27(suppl_5):v1-27. https://doi. org/10.1093/annonc/mdw326.

8. M a S, Smit EF, Lorigan P, Konduri K, Reck M, Szczesna A, et al. Phase III study of pemetrexed plus carboplatin compared with etoposide plus carboplatin in chemotherapy-naive patients with extensive-stage small-cell lung cancer. J Clin Oncol. 2009;27:4787-92. https://doi.org/10.1200/JCO. 2009.23.1548.

9. Scagliotti GV, Parikh P, von Pawel J, Biesma B, Vansteenkiste J, Manegold C, et al. Phase III study comparing cisplatin plus gemcitabine with cisplatin plus pemetrexed in chemotherapy-naive patients with advanced-stage nonsmall-cell lung cancer. J Clin Oncol. 2008;26:3543-51. https://doi.org/10. 1200/JCO.2007.15.0375.

10. Hanna N, F a S, Fossella FV, Pereira JR, De Marinis F, von Pawel J, et al. Randomized phase III trial of pemetrexed versus docetaxel in patients with non-small-cell lung cancer previously treated with chemotherapy. J Clin Oncol. 2004;22:1589-97. https://doi.org/10.1200/JCO.2004.08.163.

11. Scagliotti G, Hanna N, Fossella F, Sugarman K, Blatter J, Peterson P, et al. The differential efficacy of pemetrexed according to NSCLC histology: a review of two phase III studies. Oncologist. 2009;14:253-63. https://doi.org/10.1634/ theoncologist.2008-0232.

12. Shih C, Chen VJ, Gossett LS, Gates SB, Mackellar WC, Habeck LL, et al. LY231514, a pyrrolo [2,3-d]pyrimidine-based antifolate that inhibits multiple folate-requiring enzymes. Cancer Res. 1997;57:1116-23 http://www.ncbi.nlm. nih.gov/pubmed/9067281. Accessed 2 Oct 2017.

13. Chattopadhyay S, Moran RG, Goldman ID. Pemetrexed: biochemical and cellular pharmacology, mechanisms, and clinical applications. Mol Cancer Ther. 2007;6:404-17. https://doi.org/10.1158/1535-7163.MCT-06-0343.

14. Hanauske A-R, Eismann U, Oberschmidt O, Pospisil H, Hoffmann S, HanauskeAbel $\mathrm{H}$, et al. In vitro chemosensitivity of freshly explanted tumor cells to pemetrexed is correlated with target gene expression. Investig New Drugs. 2007;25:417-23. https:/doi.org/10.1007/s10637-007-9060-9.

15. Sigmond J, Backus HHJ, Wouters D, Temmink OH, Jansen G, Peters GJ. Induction of resistance to the multitargeted antifolate Pemetrexed (ALIMTA) in WiDr human colon cancer cells is associated with thymidylate synthase overexpression. Biochem Pharmacol. 2003;66:431-8. https://doi.org/10.1016/ S0006-2952(03)00287-9.

16. Takezawa K, Okamoto I, Okamoto W, Takeda M, Sakai K, Tsukioka S, et al. Thymidylate synthase as a determinant of pemetrexed sensitivity in nonsmall cell lung cancer. Br J Cancer. 2011;104:1594-601. https://doi.org/10. 1038/bjc.2011.129.

17. Ozasa H, Oguri T, Uemura T, Miyazaki M, Maeno K, Sato S, et al. Significance of thymidylate synthase for resistance to pemetrexed in lung cancer. Cancer Sci. 2010;101:161-6. https://doi.org/10.1111/j.1349-7006.2009.01358.x.

18. Liu Y, Yin T-J, Zhou R, Zhou S, Fan L, Zhang R-G. Expression of thymidylate synthase predicts clinical outcomes of pemetrexed-containing chemotherapy for non-small-cell lung cancer: a systemic review and meta-analysis. Cancer Chemother Pharmacol. 2013;72:1125-32. https://doi.org/10.1007/s00280-0132299-2.

19. Ceppi P, Volante M, Saviozzi S, Rapa I, Novello S, Cambieri A, et al. Squamous cell carcinoma of the lung compared with other histotypes shows higher messenger RNA and protein levels for thymidylate synthase. Cancer. 2006;107:1589-96. https://doi.org/10.1002/cncr.22208.

20. Monica V, Scagliotti GV, Ceppi P, Righi L, Cambieri A, Lo lacono M, et al. Differential thymidylate synthase expression in different variants of large-cell carcinoma of the lung. Clin Cancer Res. 2009;15:7547-52. https://doi.org/10. 1158/1078-0432.CCR-09-1641.

21. Christoph DC, Asuncion BR, Hassan B, Tran C, Maltzman JD, O'Shannessy DJ, et al. Significance of folate receptor alpha and thymidylate synthase protein expression in patients with non-small-cell lung Cancer treated with Pemetrexed. J Thorac Oncol. 2013;8:19-30. https://doi.org/10.1097/JTO. ob013e31827628ff. 
22. Wynes MW, Konopa K, Singh S, Reyna-Asuncion B, Ranger-Moore J, Sternau A, et al. Thymidylate synthase protein expression by $I H C$ and gene copy number by SISH correlate and show great variability in non-small cell lung Cancer. J Thorac Oncol. 2012;7:982-92. https://doi.org/10.1097/JTO. Ob013e31824fe95a.

23. Sun J-M, Han J, Ahn JS, Park K, Ahn M-J. Significance of thymidylate synthase and thyroid transcription factor 1 expression in patients with nonsquamous non-small cell lung cancer treated with pemetrexed-based chemotherapy. J Thorac Oncol. 2011;6:1392-9. https://doi.org/10.1097/JTO. Ob013e3182208ea8.

24. Nicolson MC, Fennell DA, Ferry D, O'Byrne K, Shah R, Potter V, et al. Thymidylate synthase expression and outcome of patients receiving Pemetrexed for advanced nonsquamous non-small-cell lung Cancer in a prospective blinded assessment phase II clinical trial. J Thorac Oncol. 2013;8:930-9. https://doi.org/10.1097/JTO.0b013e318292c500.

25. Shimizu T, Nakanishi Y, Nakagawa Y, Tsujino I, Takahashi N, Nemoto N, et al. Association between expression of thymidylate synthase, dihydrofolate reductase, and glycinamide ribonucleotide formyltransferase and efficacy of pemetrexed in advanced non-small cell lung cancer. Anticancer Res. 2012; 32:4589-96 http://www.ncbi.nlm.nih.gov/pubmed/23060591. Accessed 18 Sep 2017.

26. Hou J, Lambers M, den Hamer B, M a d B, Hoogsteden HC, Grosveld F, et al. Expression profiling-based subtyping identifies novel non-small cell lung cancer subgroups and implicates putative resistance to pemetrexed therapy. J Thorac Oncol. 2012;7:105-14. https://doi.org/10.1097/JTO. Ob013e3182352a45.

27. Tusher VG, Tibshirani R, Chu G. Significance analysis of microarrays applied to the ionizing radiation response. Proc Natl Acad Sci U S A. 2001;98:5116-21. https://doi.org/10.1073/pnas.091062498.

28. McShane LM, Altman DG, Sauerbrei W, Taube SE, Gion M, Clark GM. Reporting recommendations for tumor marker prognostic studies (REMARK). JNCI J Natl Cancer Inst. 2005;97:1180-4. https://doi.org/10.1093/jnci/dji237.

29. Landis JR, Koch GG. The measurement of observer agreement for categorical data. Biometrics. 1977;33:159-74 http://www.ncbi.nlm.nih.gov/pubmed/ 843571. Accessed 3 Oct 2017.

30. Hosmer DW, Lemeshow S, Sturdivant RX. Applied logistic regression, 3rd edition: John Wiley and Sons Ltd, New York, April 2013. https://www.wiley. com/en-us/Applied+Logistic+Regression,+3rd+Edition-p-9780470582473. Accessed 13 May 2018

31. Schneider MA, Christopoulos P, Muley T, Warth A, Klingmueller U, Thomas M, et al. AURKA, DLGAP5, TPX2, KIF11 and CKAP5: five specific mitosis-associated genes correlate with poor prognosis for non-small cell lung cancer patients. Int J Oncol. 2017;50:365-72. https://doi.org/10. 3892/ijo.2017.3834.

32. Martens-de Kemp SR, Nagel R, Stigter-van Walsum $M$, van der Meulen $\mathbb{H}_{\text {, }}$ van Beusechem WW, Braakhuis BJM, et al. Functional genetic screens identify genes essential for tumor cell survival in head and neck and lung cancer. Clin Cancer Res. 2013;19:1994-2003. https://doi.org/10.1158/1078-0432.CCR12-2539.

33. Behrens C, Solis LM, Lin H, Yuan P, Tang X, Kadara H, et al. EZH2 protein expression associates with the early pathogenesis, tumor progression, and prognosis of non-small cell lung carcinoma. Clin Cancer Res. 2013;19:6556-65. https://doi.org/10.1158/1078-0432.CCR-12-3946.

34. Huqun $R$, Ishikawa $R$, Zhang J, Miyazawa $H$, Goto $Y$, Shimizu $Y$, et al. Enhancer of zeste homolog 2 is a novel prognostic biomarker in nonsmall cell lung cancer. Cancer. 2012;118:1599-606. https://doi.org/10. 1002/cncr.26441.

35. Reijm EA, Timmermans AM, Look MP, Meijer-van Gelder ME, Stobbe CK, van Deurzen $\mathrm{CHM}$, et al. High protein expression of $\mathrm{EZH} 2$ is related to unfavorable outcome to tamoxifen in metastatic breast cancer. Ann Oncol. 2014;25:2185-90. https://doi.org/10.1093/annonc/mdu391.

36. van Kruijsdijk RCM, Visseren FLJ, Boni L, Groen HJM, Dingemans AMC, Aerts JGJV, et al. Pemetrexed plus carboplatin versus pemetrexed in pretreated patients with advanced non-squamous non-small-cell lung cancer: treating the right patients based on individualized treatment effect prediction. Ann Oncol. 2016;27:1280-6. https://doi.org/10.1093/ annonc/mdw154

37. Gonen N, Assaraf YG. Antifolates in cancer therapy: structure, activity and mechanisms of drug resistance. Drug Resist Updat. 2012;15:183-210. https:// doi.org/10.1016/j.drup.2012.07.002.
38. Shaw a T, Varghese a M, Solomon BJ, Costa DB, Novello S, Mino-Kenudson $M$, et al. Pemetrexed-based chemotherapy in patients with advanced, ALKpositive non-small cell lung cancer. Ann Oncol. 2013;24:59-66. https://doi. org/10.1093/annonc/mds242.

39. Park S, Ahn B-C, Lim SW, Sun J-M, Kim HR, Hong MH, et al. Characteristics and outcome of ROS1-positive non-small cell lung Cancer patients in routine clinical practice. J Thorac Oncol. 2018;13:1373-82. https://doi.org/10. 1016/j.jtho.2018.05.026.

\section{Ready to submit your research? Choose BMC and benefit from:}

- fast, convenient online submission

- thorough peer review by experienced researchers in your field

- rapid publication on acceptance

- support for research data, including large and complex data types

- gold Open Access which fosters wider collaboration and increased citations

- maximum visibility for your research: over $100 \mathrm{M}$ website views per year

At $\mathrm{BMC}$, research is always in progress.

Learn more biomedcentral.com/submissions 